\title{
Real-time tunability of chip-based light source enabled by microfluidic mixing
}

\author{
Olsen, Brian Bilenberg; Rasmussen, Torben; Balslev, Søren; Kristensen, Anders
}

Published in:

Journal of Applied Physics

Link to article, DOI:

$10.1063 / 1.2163011$

Publication date:

2006

Document Version

Publisher's PDF, also known as Version of record

Link back to DTU Orbit

Citation (APA):

Olsen, B. B., Rasmussen, T., Balslev, S., \& Kristensen, A. (2006). Real-time tunability of chip-based light source enabled by microfluidic mixing. Journal of Applied Physics, 99(2), 023102. https://doi.org/10.1063/1.2163011

\section{General rights}

Copyright and moral rights for the publications made accessible in the public portal are retained by the authors and/or other copyright owners and it is a condition of accessing publications that users recognise and abide by the legal requirements associated with these rights.

- Users may download and print one copy of any publication from the public portal for the purpose of private study or research.

- You may not further distribute the material or use it for any profit-making activity or commercial gain

- You may freely distribute the URL identifying the publication in the public portal 


\title{
Real-time tunability of chip-based light source enabled by microfluidic mixing
}

\author{
Brian Bilenberg, Torben Rasmussen, Søren Balslev, and Anders Kristensen ${ }^{\text {a) }}$ \\ MIC-Department of Micro and Nanotechnology, Technical University of Denmark, \\ Building 345 East, Ørsteds Plads, DK-2800 Kongens Lyngby, Denmark
}

(Received 20 April 2005; accepted 2 December 2005; published online 23 January 2006)

\begin{abstract}
We demonstrate real-time tunability of a chip-based liquid light source enabled by microfluidic mixing. The mixer and light source are fabricated in SU-8 which is suitable for integration in SU-8-based laboratory-on-a-chip microsystems. The tunability of the light source is achieved by changing the concentration of rhodamine $6 \mathrm{G}$ dye inside two integrated vertical resonators, since both the refractive index and the gain profile are influenced by the dye concentration. The effect on the refractive index and the gain profile of rhodamine $6 \mathrm{G}$ in ethanol is investigated and the continuous tuning of the laser output wavelength is demonstrated using an ethanolic rhodamine $6 \mathrm{G}$ solution of $2 \times 10^{-2} \mathrm{~mol} / 1$ mixed with pure ethanol. This yields rhodamine $6 \mathrm{G}$ concentrations from $5 \times 10^{-3}$ to $1.5 \times 10^{-2} \mathrm{~mol} / 1$ inside the laser resonators and a wavelength change of $10 \mathrm{~nm}$ with a response time of 110 s. () 2006 American Institute of Physics. [DOI: 10.1063/1.2163011]
\end{abstract}

\section{INTRODUCTION}

The concept of using liquid media to add or improve the functionality of optical devices has been widely pursued. Liquid core waveguides ${ }^{1}$ have been used to increase the interaction length in sensing applications. The introduction of the integrated laboratory on a chip ${ }^{2,3}$ with the capabilities of fast and precise liquid flow control and mixing has opened paths for the field of "optofluidics". 4,5 The ability to precisely control a liquid medium is essential to the control of optical properties, such as refractive index and optical gain media concentration. Optofluidics offers the ability to adjust these parameters in real-time through, e.g., microfluidic mixing of liquids in a precise and predictable fashion, as we will demonstrate in this paper. The characteristics of liquid dye lasers are determined by the dye concentration and the physical dimensions of the laser resonator. Hence, the properties of the light source can be altered by adjusting these parameters, enabling tunability of the output wavelength. ${ }^{6,7}$ We demonstrate a microfluidic mixer (see Fig. 1) with the capability to mix two liquids in situ through diffusion mixing and show how this can be used to alter the optical properties of a microresonator using the organic dye, rhodamine $6 \mathrm{G}$, as active medium. By diluting an ethanolic rhodamine $6 \mathrm{G}$ solution in real time the dye solution can be adjusted, enabling both concentration tuning and refractive index tuning within the laser resonators. Two types of vertical cavities have been implemented to provide easy readout of the optical properties of the dye medium - a Fabry-Pérot resonator defined by vertically aligned opposing mirrors ${ }^{8}$ and a dielectric resonator relying on the refractive index changes to the surrounding cladding.

The response of the system is investigated to determine how readily the output wavelength can be altered, as the relative flow rate of an ethanolic rhodamine $6 \mathrm{G}$ solution and a pure ethanolic solution is adjusted.

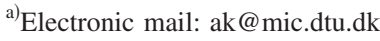

\section{TUNING MECHANISM}

The lasing wavelength of a Fabry-Pérot dye laser depends on the gain profile of the dye, the dye concentration, ${ }^{6}$ and the refractive index inside the laser cavity, apart from the Fabry-Pérot cavity modes themselves. For macroscopic organic dye laser systems it is well known that the concentration of dye in the liquid solution must be kept at a certain value to ensure reproducible functionality of the laser system, since both the refractive index ${ }^{9}$ and the gain profile ${ }^{6}$ are influenced by the dye concentration. In a microsystem that allows fast and precise on-chip mixing of fluids, ${ }^{2}$ this can be used to control and tune the refractive index and the gain profile of the dye solution to achieve real-time wavelength tunability of a laser device.

The concentration dependency of the gain profile is caused by the Stokes shift. Figure 2 illustrates the dynamics of the dye gain during the increase of optical pumping of an ensemble of dye molecules. With increasing pump power, the net absorption will decrease and the net gain will increase according to the population inversion of the electronic states of the molecules. The total gain is a result of both the net absorption and the net gain, and will move spectrally with

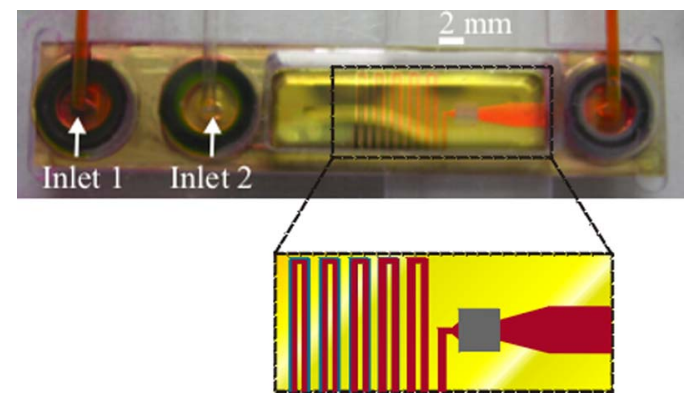

FIG. 1. Top: photograph of the tunable dye laser mounted in a sample holder fitted with inlet and outlet connections. An ethanolic rhodamine 6G solution (inlet 1) is mixed with pure ethanol (inlet 2). Bottom: the sketch shows the meander mixing channel connected to the laser resonator. 


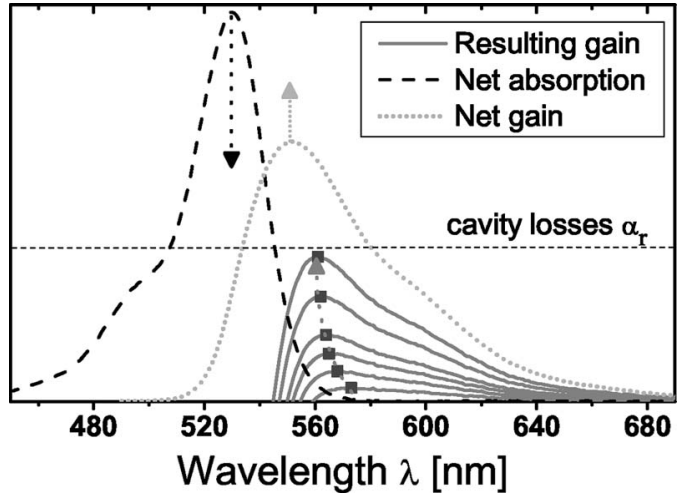

FIG. 2. Illustration of the dynamics of the dye gain during the increase of optical pumping.

increasing inversion. In a laser the total gain will increase with increased pumping power until the lasing threshold, where the total gain will be clamped. Since the amount of dye molecules determines at what rate the total gain will rise with increasing inversion (or pumping) the wavelength where the system will reach lasing threshold depends on the dye concentration.

The refractive index in the laser resonator, together with the mirrors and the properties of the dielectric medium, determines the position of the Fabry-Pérot cavity modes according to the phase-matching condition

$$
2 \pi q=2 k d n
$$

where $q=1,2,3, \ldots, k=2 \pi / \lambda, d$ is the Fabry-Pérot mirror distance, and $n$ is the refractive index between the mirrors. Obviously the change in wavelength is $d \lambda / d n=2 d / q$.

\section{DESIGN}

In order to investigate the achievable tunability of the presented tunable microfluidic light source, a vertical resonator design is used. Two different resonator configurations have been investigated: a Fabry-Pérot cavity formed inside a microfluidic channel by two parallel planar metallic mirrors on the floor and roof of the channel, ${ }^{8}$ and the same resonator arrangement without a metallic top mirror (see Fig. 3). The metallic mirror on the floor consists of $10 \mathrm{~nm}$ chromium and $150 \mathrm{~nm}$ gold (reflectance $=0.83$, transmittance $=0$, and absorbance $=0.17$ at $\lambda=570 \mathrm{~nm}$ ), while the semitransparent metallic mirror on the roof consists of $5 \mathrm{~nm}$ chromium and $40 \mathrm{~nm}$ gold (reflectance $=0.72$, transmittance $=0.06$, and absorbance $=0.22$ at $\lambda=570 \mathrm{~nm}$ ). Thus, lasing light is emitted through the semitransparent mirror in a direction perpendicular to the mirrors and the chip plane. The height of the microfluidic channel is $7.5 \mu \mathrm{m}$, as defined by the SU-8 layer. An ethanolic solution of the organic dye, in this case rhodamine $6 \mathrm{G}$, flows through the resonator while it is optically pumped through the glass lid at an angle of approximately $45^{\circ}$ to normal using a frequency-doubled Nd:YAG (yttrium aluminum garnet) laser $(\lambda=532 \mathrm{~nm})$. Diluted solutions of rhodamine $6 \mathrm{G}$ are used as gain medium, so the refractive index inside the laser resonator is only slightly above that of pure ethanol, $n=1.36$. The $2.6 \mu \mathrm{m}$ polymethylmehtacrylate (PMMA) layer has a refractive index of 1.49 . Hence, the mode distance is approximately $11.5 \mathrm{~nm}$ for the opposing metallic mirror cavity and $0.2 \mathrm{~nm}$ for the resonator without a metallic top mirror at wavelengths around $\lambda=570 \mathrm{~nm}$, since the top surface of the glass lid becomes the major reflector with a reflectance of 0.04 .

\section{Top view}

a)

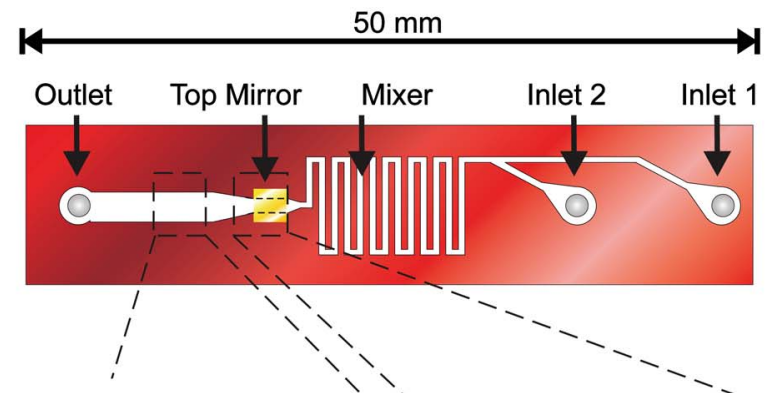

Cross section

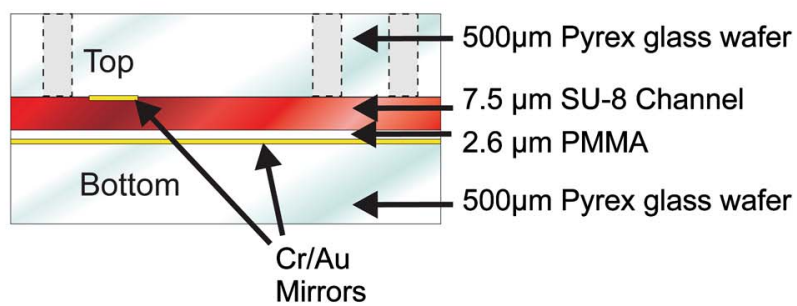

b)

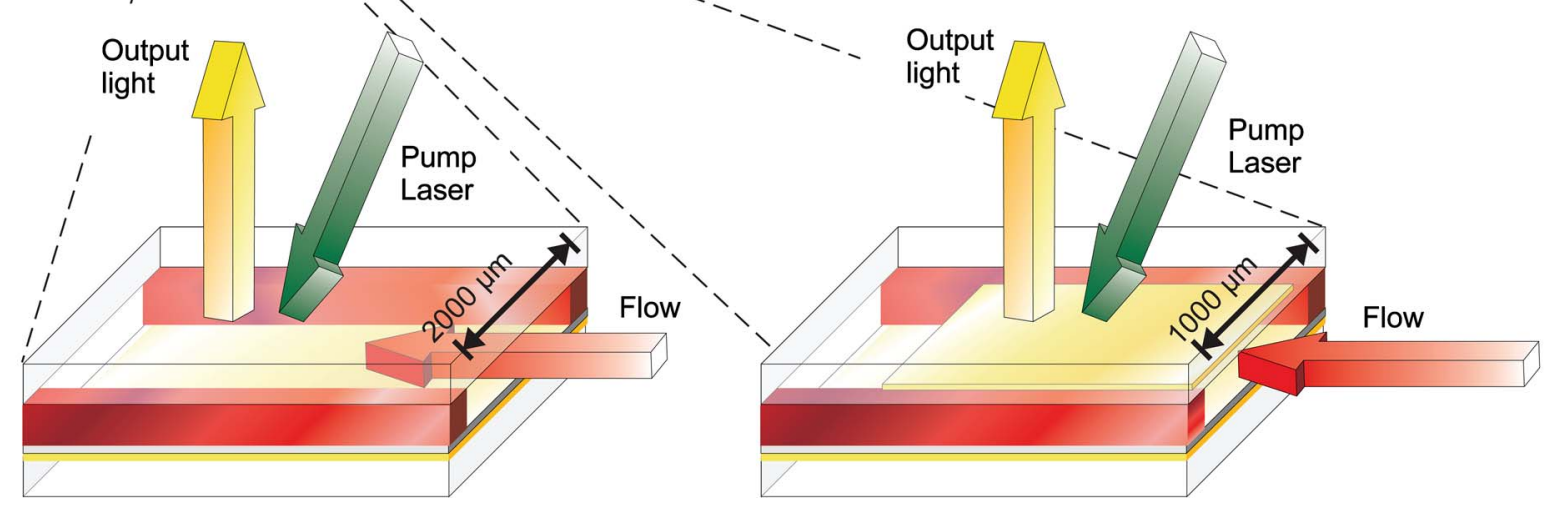

FIG. 3. (a) Microfluidic mixer and resonator layout with a 200- $\mu \mathrm{m}$-wide mixer channel. Top wafer: $500 \mu \mathrm{m}$ Pyrex, $5 \mathrm{~nm}$ chromium, and $40 \mathrm{~nm}$ gold; $7.5 \mu \mathrm{m}$ SU-8 defining the mixer channels and drilled holes for inlets and outlet. Bottom wafer: $500 \mu \mathrm{m}$ Pyrex, $10 \mathrm{~nm}$ chromium, and $150 \mathrm{~nm}$ gold; $2.6 \mu \mathrm{m}$ PMMA. (b) The two areas used for pumping —one with a semitransparent metallic top mirror and one without. 
The vertical emitting laser resonator is integrated with a microfluidic mixer with two inlets. This way the dye concentration in the laser resonator is controlled by the relative flow rate between an ethanolic solution of rhodamine $6 \mathrm{G}$ and pure ethanol in the mixer. Due to the small dimensions, the flow in the mixer is laminar (Reynolds number of 0.2) and mixing is limited by molecular diffusion across the channel. Different more efficient mixer layouts that reduce the mixing time substantially have been proposed. ${ }^{10,11}$ However, the implementation of these layouts in a planar processing scheme is troublesome. Hence, a simple diffusive mixer was chosen for this demonstration of the tuning principle of a microfluidic dye laser. Furthermore, the external delays in the syringe pumps used to operate the device could be minimized by integrating pumps, as proposed in Ref. 12.

The channel height is identical to that of the laser resonator, $7.5 \mu \mathrm{m}$. The diffusion constant of rhodamine $6 \mathrm{G}$ in ethanol is estimated to be $1 \times 10^{-6} \mathrm{~cm}^{2} / \mathrm{s}$ from the StokesEinstein relation. ${ }^{13,14}$ Due to the pressure-driven laminar flow in the fluidic channel the velocity profile will be parabolic. In the estimation of the mixing time, the parabolic velocity profile in the vertical direction can be neglected. The height of the fluidic channel is only $7.5 \mu \mathrm{m}$ and it takes only $0.28 \mathrm{~s}$ for a rhodamine $6 \mathrm{G}$ molecule to diffuse from the bottom to the top of the channel, estimated by the EinsteinSchmoluchowski relation. Therefore a one-dimensional model, only considering the velocity profile in the lateral direction, is used to describe the flow in the mixer channel. From this simplified model, one finds that the foremost part of the interface between the old and the new solutions will reach the optical resonator after $76 \mathrm{~s}$ when the device is operated at a flow rate of $5 \mu \mathrm{l} / \mathrm{h}$ and the dielectric resonator is positioned, as shown in Fig. 3(b). If the pure ethanol solution is turned off, a front of rhodamine $6 \mathrm{G}$ solution will be propagating with a flow rate of $5 \mu \mathrm{l} / \mathrm{h}$ and the first rhodamine $6 \mathrm{G}$ molecules will reach the resonator in $76 \mathrm{~s}$, at which point one should expect to see a change in the wavelength of the output light. Averaging the velocity field across the mixer channel, the rhodamine is predicted to arrive at the dielectric resonator after $114 \mathrm{~s}$ at a flow rate of $5 \mu \mathrm{l} / \mathrm{h}$.

\section{FABRICATION}

The tunable microfludic light source is fabricated on a Borofloat glass substrate. Prior to fabrication the glass substrate is cleaned in Triton X100 soap and 7-up etch (sulfuric acid and ammoniumperoxodisulfate at $80^{\circ} \mathrm{C}$ ) before dehydration for $12 \mathrm{~h}$ in an oven at $220^{\circ} \mathrm{C}$. The bottom metallic mirror is deposited by vapor deposition before spinning on a 7.5- $\mu$ m-thick layer of SU-8 photoresist (MicroChem SU-8 10) at a spin speed of $3000 \mathrm{rpm}$ and prebaking on a hot plate $\left(65^{\circ} \mathrm{C}\right.$ for $2 \mathrm{~min}$ and $90{ }^{\circ} \mathrm{C}$ for $2 \mathrm{~min}$ ). The SU- 8 photoresist is patterned by UV $(\lambda=365 \mathrm{~nm})$ lithography (dose: $\left.490 \mathrm{~mJ} / \mathrm{cm}^{2}\right)$, cross-linked by baking on a hot plate $\left(65^{\circ} \mathrm{C}\right.$ for $2 \mathrm{~min}$ and $90{ }^{\circ} \mathrm{C}$ for $15 \mathrm{~min}$ ) and developed in propylene glycol monomethyl ether acetate (PGMEA) (Microchem) for $4 \mathrm{~min}$. The top metallic mirror is defined on a clean glass lid by UV lithography and lift-off. A layer of 2.6- $\mu \mathrm{m}$-thick 950 K PMMA is used to seal the SU-8 structures with the glass

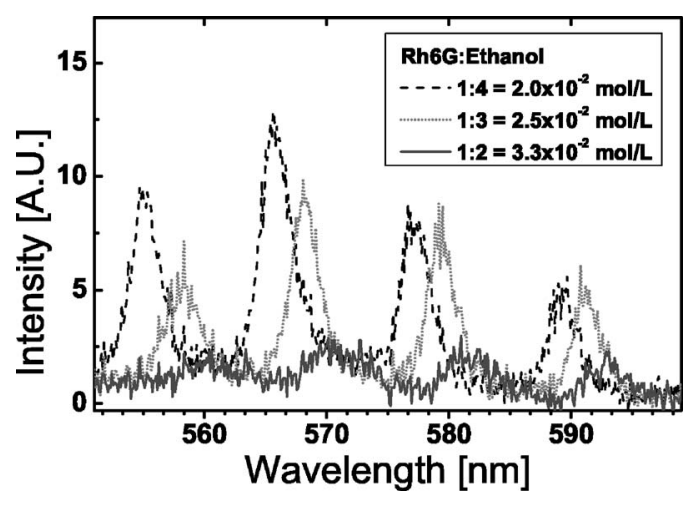

FIG. 4. Emission spectrum from the Fabry-Pérot cavity defined by two parallel metallic mirrors with varying rhodamine $6 \mathrm{G}$ concentrations. The spectrum is obtained by pumping the cavity with $58 \mu \mathrm{J} / \mathrm{mm}^{2}$. To avoid evaporation of the $\mathrm{Cr} / \mathrm{Au}$ mirrors, the pump energy should be kept below $80 \mu \mathrm{J} / \mathrm{mm}^{2}$. The Fabry-Pérot modes are tuned continuously $4 \mathrm{~nm}$ by adjusting the relative inlet flow rates of ethanolic rhodamine $6 \mathrm{G}$ solution $\left(10^{-1} \mathrm{~mol} / \mathrm{l}\right)$ and pure ethanol.

lid. ${ }^{15}$ Finally, the device is diced into $10 \times 50 \mathrm{~mm}^{2}$ chips and inlet and outlet holes are drilled through the top glass lid. Figure 1 shows an optical image of a final device mounted in a holder fitted with fluidic connections. Scanning electron microscopy (SEM) inspections of a cleaved device verifies that the height of the SU-8 layer is $7.5 \mu \mathrm{m}$ and that the height of the PMMA layer is $2.6 \mu \mathrm{m}$.

\section{RESULTS}

The laser device is operated with different concentrations of rhodamine $6 \mathrm{G}$ dye in ethanol mixed with pure ethanol at different flow ratios, yielding controlled rhodamine 6G concentrations inside the optical cavities. The cavities are optically pumped with a pulsed (5 ns pulse length at a rate of $10 \mathrm{~Hz})$ frequency-doubled Nd:YAG laser $(\lambda=532 \mathrm{~nm})$ at an angle of incidence of approximately $45^{\circ}$ and the dye laser output is collected by an optical fiber positioned $1 \mathrm{~cm}$ vertically above the cavities.

The Fabry-Pérot cavity defined by metallic mirrors is used to investigate the influence of rhodamine $6 \mathrm{G}$ concentration on the refractive index inside the cavity. The cavity has not been used above lasing threshold because the semitransparent metallic top mirror degrades at larger pumping intensities. From a $1 \times 10^{-2} \mathrm{~mol} / 1$ rhodamine $6 \mathrm{G}$ solution and pure ethanol, the rhodamine $6 \mathrm{G}$ concentration inside the laser cavity is changed between $2.0 \times 10^{-2}$ and 3.3 $\times 10^{-2} \mathrm{~mol} / 1$ by mixing. This way the Fabry-Pérot cavity modes are tuned $4 \mathrm{~nm}$, as seen in Fig. 4. We attribute this tuning to the change of refractive index due to the presence of dye in the ethanol.

According to the phase condition (1) for the cavity defined by metallic mirrors, $q \lambda=2\left(n_{1} L_{1}+n_{2} L_{2}\right)$, where $n_{1}$ and $n_{2}$ are the refractive indices of the dye solution and the sealing PMMA layer (1.49), respectively, $L_{1}$ and $L_{2}$ are the thicknesses of the dye solution $(7.5 \mu \mathrm{m})$ and the PMMA layer $(2.6 \mu \mathrm{m}$ ), and $q$ is the mode number (found to be 49), the measured tuning range, $\Delta \lambda=4 \mathrm{~nm}$, corresponds to a refractive index change inside the cavity of $\Delta n_{1}=\Delta \lambda \times q / 2 L_{1}$ $=0.013$, resulting in a $\Delta n_{1} / \Delta c$ of $11 / \mathrm{mol}\left(2.2 \times 10^{-3} \mathrm{l} / \mathrm{g}\right)$. 


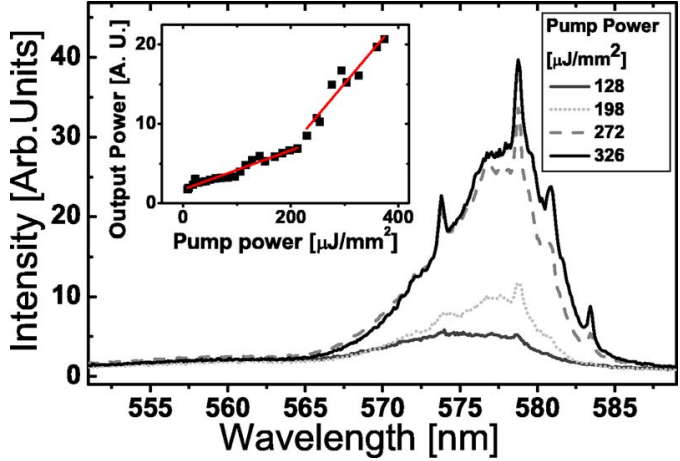

FIG. 5. Emission spectrum from the microresonator fluidic dye laser with $1 \times 10^{-2} \mathrm{~mol} / 1$ rhodamine $6 \mathrm{G}$ solution as the pump power is gradually increased. Pumping is carried out in the open channel near the outlet and multimode lasing is observed at approximately $110 \mu \mathrm{J} / \mathrm{mm}^{2}$. The rhodamine concentration is achieved by mixing a $2 \times 10^{-2} \mathrm{~mol} / 1$ rhodamine solution of 1:1 with pure ethanol using the integrated mixer. Inset: average dye output power (arbitrary units) estimated by integrating the output spectra showing the lasing threshold.

This value is comparable to the value in Ref. 9 for rhodamine-B in ethanol at $570 \mathrm{~nm}$, which was found to $0.8 \mathrm{l} / \mathrm{mol}$. This large value is due to the anomalous dispersion curve around the molecular resonance. Furthermore, the spectrum in Fig. 4 shows that the mode spacing is approximately $11.5 \mathrm{~nm}$, as expected from the design considerations.

When pumping the metallic mirror resonator, the $532 \mathrm{~nm}$ pump light will be absorbed by the organic dye after a few micrometers due to its low intensity and the relatively high concentration of dye. The rest of the dye will be absorbing and lead to a lower cavity-enhanced output signal. In addition the quantum yield of the organic dye decreases with increasing concentration. ${ }^{16}$ We ascribe the lowered output signal for increased dye concentration (Fig. 4) to these mechanisms.

The finesse, $f$, of the resonator containing the dye solution is determined to be 3.8 from the data in Fig. 4 by applying a multiple Lorentzian peak fit and using $f$ $=$ FSR $/$ FWHM, where FSR is the free spectral range and FWHM is the full width at half maximum of the individual peaks. Using the absorption cross section for rhodamine $6 \mathrm{G}$ and the metallic mirror reflectivities, the expected finesse is 4.1.

The dielectric resonator is used to investigate rhodamine $6 \mathrm{G}$ dye concentration tuning and the response time of the tunable device. The inset in Fig. 5 shows the threshold curve for the dielectric resonator as the pumping energy is gradually increased. The onset of lasing is around $110 \mu \mathrm{J} / \mathrm{mm}^{2}$. From a $2 \times 10^{-2} \mathrm{~mol} / 1$ rhodamine $6 \mathrm{G}$ solution and pure ethanol, the rhodamine $6 \mathrm{G}$ concentration inside the laser resonator is changed between $5.6 \times 10^{-3}$ and $2 \times 10^{-2} \mathrm{~mol} / \mathrm{l}$. As seen from the laser output spectra in Fig. 6, this results in a continuous wavelength tuning range of $10 \mathrm{~nm}$ between 573 and $583 \mathrm{~nm}$. The mode spacing of the dielectric cavity is 0.2 $\mathrm{nm}$ and therefore not resolved in our experiments. Hence, a shift in the cavity modes cannot account for the shift of the multimode peaks. We attribute the spikes on the spectra to self-interference of the pumping laser beam as it is reflected from the bottom mirror creating a volume grating enhancing

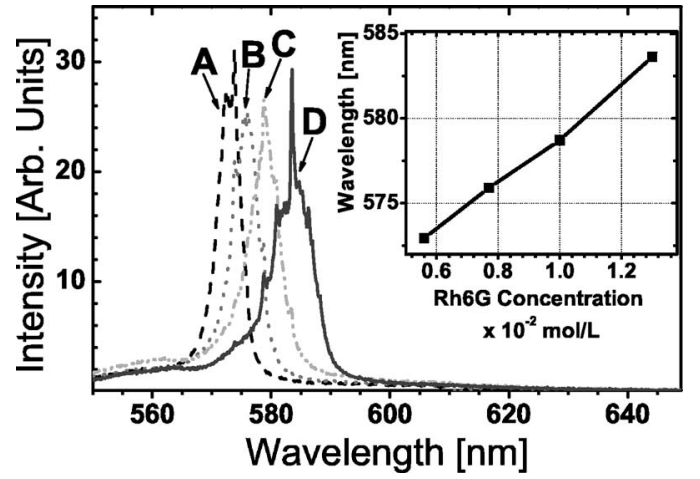

FIG. 6. Emission spectrum from the microresonator fluidic dye laser with varying rhodamine $6 \mathrm{G}$ concentrations. The spectrum is obtained by pumping the resonator in the open channel near the outlet at $111 \mu \mathrm{J} / \mathrm{mm}^{2}$ (except the highest concentration of $1.3 \times 10^{-2} \mathrm{~mol} / \mathrm{l}$ ). The peak position is moved continuously from 573 to $583 \mathrm{~nm}$ by adjusting the relative flow rate of ethanol and rhodamine $6 \mathrm{G}$ solution. The rhodamine solution is $2 \times 10^{-2} \mathrm{~mol} / 1$. (A: $5.6 \times 10^{-3} \mathrm{~mol} / 1, \quad$ B: $7.7 \times 10^{-3} \mathrm{~mol} / 1, \quad$ C: $1.0 \times 10^{-2} \mathrm{~mol} / 1$, and D: 1.3 $\left.\times 10^{-2} \mathrm{~mol} / 1\right)$.

some modes in the resonator. The inset in Fig. 6 shows a nearly linear relationship between concentration and lasing wavelength in this range. The position of the peaks is determined from the center wavelength at half maximum height of the peaks.

In order to measure the response time of the device, a steady flow of $2.5 \mu \mathrm{l} / \mathrm{h}$ rhodamine $6 \mathrm{G}$ ethanolic solution $\left(2 \times 10^{-2} \mathrm{~mol} / \mathrm{l}\right)$ and $7.5 \mu \mathrm{l} / \mathrm{h}$ pure ethanol is injected, yielding an effective concentration of $5 \times 10^{-3} \mathrm{~mol} / 1$ inside the resonator after mixing. The flow of the pure ethanol is turned off at the same time as the flow rate of the rhodamine $6 \mathrm{G}$ solution is increased to $5 \mu \mathrm{l} / \mathrm{h}$. This will eventually result in a rhodamine $6 \mathrm{G}$ concentration of $2 \times 10^{-2} \mathrm{~mol} / 1$ inside the laser resonator. To monitor the time evolution of the concentration inside the laser resonator, lasing output spectra are recorded continuously until the change in lasing wavelength saturates, see Fig. 7. From Fig. 7, it is seen that the concentration inside the laser resonator begins to increase after $60 \mathrm{~s}$ and saturates after $110 \mathrm{~s}$. The onset of change in the lasing wavelength corresponds with the expected time of $76 \mathrm{~s}$ from the design considerations. The uncertainty of the position of

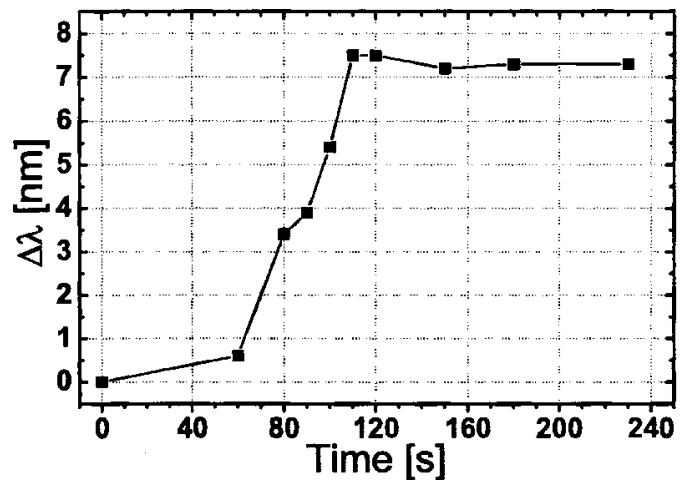

FIG. 7. Response curve for the dielectric resonator as the inlet flow rates are changed from $2.5 \mu \mathrm{l} / \mathrm{h}$ rhodamine $6 \mathrm{G}\left(2 \times 10^{-2} \mathrm{~mol} / \mathrm{l}\right)$ and $7.5 \mu \mathrm{l} / \mathrm{h}$ pure ethanol to $5 \mu \mathrm{l} / \mathrm{h}$ rhodamine $6 \mathrm{G}$ and $0 \mu \mathrm{l} / \mathrm{h}$ ethanol. This corresponds to a concentration shift from $5 \times 10^{-3}$ to $2 \times 10^{-2} \mathrm{~mol} / 1$. The graph shows the increase in the lasing wavelength $(\Delta \lambda)$ as a function of the elapsed time in seconds. The relative flow rate is changed at $t=0$. 
the pump-laser spot (diameter of $1 \mathrm{~mm}$ ) is $\pm 1 \mathrm{~mm}$ from the ideal position, corresponding to an uncertainty in the response time of $\pm 10.8 \mathrm{~s}$.

\section{CONCLUSION}

Real-time tunability of a chip-based liquid light source enabled by microfluidic mixing has been demonstrated. The tunability of the output light was achieved by changing the concentration of rhodamine $6 \mathrm{G}$ inside the optical cavities by means of an integrated microfluidic mixer. Continuous tuning of the laser output wavelength was demonstrated using an ethanolic rhodamine $6 \mathrm{G}$ solution of $2 \times 10^{-2} \mathrm{~mol} / 1 \mathrm{mixed}$ with pure ethanol. The response time of the optofluidic device was measured to be $110 \mathrm{~s}$. This could be improved by means of optimized mixer designs. Furthermore, the refractive index change of ethanol as a function of rhodamine $6 \mathrm{G}$ concentration has been investigated. This shows that a change in rhodamine $6 \mathrm{G}$ concentration from $3.3 \times 10^{-2}$ to $2.0 \times 10^{-2} \mathrm{~mol} / 1$ results in a refractive index change of 0.013 , corresponding to $\Delta n / \Delta c=11 / \mathrm{mol}$.

\section{ACKNOWLEDGMENTS}

We thank Jörg P. Kutter for valuable discussions. The work was supported by the Danish Technical Research Council (STVF, Grant No. 26-02-0064). The partial support of the EC-funded project $\mathrm{NaPa}$ (Contract No. NMP4-CT-
2003-500120) is gratefully acknowledged. The content of this work is the sole responsibility of the authors.

${ }^{1}$ P. Dress and H. Franke, Rev. Sci. Instrum. 68, 2167 (1996).

${ }^{2}$ A. Manz, N. Graber, and H. M. Widmer, Sens. Actuators B 1, 244 (1990).

${ }^{3}$ E. Verpoorte and N. F. D. Roji, Proc. IEEE 91, 930 (2003).

${ }^{4}$ D. V. Vezenov, B. T. Mayers, D. B. Wolfe, and G. M. Whitesides, Appl. Phys. Lett. 86, 041104 (2005).

${ }^{5}$ D. B. Wolfe, R. S. Conroy, P. Garstecki, B. T. Mayers, M. A. Fischbach, K. E. Paul, M. Prentiss, and G. M. Whitesides, Proc. Natl. Acad. Sci. U.S.A. 101, 12434 (2004).

${ }^{6}$ F. P. Schäfer, Dye Lasers (Springer, Berlin, 1973).

${ }^{7}$ O. G. Peterson, J. P. Webb, W. C. McColgin, and J. H. Eberly, J. Appl. Phys. 42, 1917 (1971).

${ }^{8}$ B. Helbo, A. Kristensen, and A. Menon, J. Micromech. Microeng. 13, 307 (2003).

${ }^{9}$ M. A. Ali, J. Moghaddasi, and S. A. Ahmed, J. Opt. Soc. Am. B 8, 1807 (1991).

${ }^{10}$ A. D. Stroock, S. K. W. Dertinger, A. Ajdari, I. Mezic, H. A. Stone, and G. M. Whitesides, Science 295, 647 (2002).

${ }^{11}$ R. H. Liu, M. A. Stremler, K. V. Sharp, M. G. Olsen, J. G. Santiago, R. J. Adrian, H. Aref, and D. J. Beebe, J. Microelectromech. Syst. 9, 190 (2000).

${ }^{12}$ S. Balslev, N. Roxhed, P. Griss, G. Stemme, and A. Kristensen, Proceedings of MicroTAS, 2004 (unpublished), Vol. 2, p. 375.

${ }^{13}$ J. Schuster, F. Cichos, J. Wrachtrup, and C. von Borczyskowski, Single Mol. 1, 299 (2000).

${ }^{14}$ D. S. Ko, M. Sauer, S. Nord, R. Müller, and J. Wolfrum, Chem. Phys. Lett. 269, 54 (1997).

${ }^{15}$ B. Bilenberg, T. Nielsen, B. Clausen, and A. Kristensen, J. Micromech. Microeng. 14, 814 (2004).

${ }^{16}$ C. Bojarski and E. Grabowska, Acta Phys. Pol. A 60, 397 (1981). 